\title{
Exoskeleton for post-stroke recovery of ambulation (ExStRA): study protocol for a mixed-methods study investigating the efficacy and acceptance of an exoskeleton- based physical therapy program during stroke inpatient rehabilitation
}

Dennis R. Louie ${ }^{1,2}$, William B. Mortenson ${ }^{2,3}$, Melanie Durocher ${ }^{4}$, Robert Teasell ${ }^{5,6,7}$, Jennifer Yao ${ }^{8,9}$ and Janice J. Eng ${ }^{10^{*}}$ (i)

\begin{abstract}
Background: The ability to walk is commonly reported as a top rehabilitation priority for individuals after a stroke. However, not all individuals with stroke are able to practice walking, especially those who require more assistance from their therapist to do so. Powered robotic exoskeletons are a new generation of roboticassisted gait training devices, designed to assist lower extremity movement to allow repetitious overground walking practice. To date, minimal research has been conducted on the use of an exoskeleton for gait rehabilitation after stroke. The following research protocol aims to evaluate the efficacy and acceptability, and thus adoptability, of an exoskeleton-based gait rehabilitation program for individuals with stroke.

Methods: This research protocol describes a prospective, multi-center, mixed-methods study comprised of a randomized controlled trial and a nested qualitative study. Forty adults with subacute stroke will be recruited from three inpatient rehabilitation hospitals and randomized to receive either the exoskeleton-based gait rehabilitation program or usual physical therapy care. The primary outcome measure is the Functional Ambulation Category at post-intervention, and secondary outcomes include motor recovery, functional mobility, cognitive, and quality-of-life measures. Outcome data will be collected at baseline, post-intervention, and at 6 months. The qualitative component will explore the experience and acceptability of using a powered robotic exoskeleton for stroke rehabilitation from the point of view of individuals with stroke and physical therapists. Semi-structured interviews will be conducted with participants who receive the exoskeleton intervention, and with the therapists who provide the intervention. Qualitative data will be analyzed using interpretive description.

(Continued on next page)
\end{abstract}

\footnotetext{
* Correspondence: janice.eng@ubc.ca

${ }^{10}$ Department of Physical Therapy, Faculty of Medicine, University of British

Columbia, 212-2177 Wesbrook Mall, Vancouver, BC V6T 1Z3, Canada

Full list of author information is available at the end of the article
} 
(Continued from previous page)

Discussion: This study will be the first mixed-methods study examining the adoptability of exoskeleton-based rehabilitation for individuals with stroke. It will provide valuable information regarding the efficacy of exoskeletonbased training for walking recovery and will shed light on how physical therapists and patients with stroke perceive the device. The findings will help guide the integration of robotic exoskeletons into clinical practice.

Trial registration: NCT02995265 (clinicaltrials.gov), Registered 16 December 2016.

Keywords: Stroke, Rehabilitation, Exoskeleton, Walking, Clinical trial

\section{Background}

Stroke is a leading cause of adult disability, often resulting in hemiparesis, altered sensation, incoordination, cognitive changes, and speech disturbances $[1,2]$. With improved detection and medical treatment of stroke, the prevalence of individuals living with such effects of stroke is continually increasing at the national and global scale [2-4]. A major factor associated with long-term disability after stroke is the ability to walk independently [5-8], and is often cited as a goal by individuals with stroke $[9,10]$. However, nearly half of individuals with stroke do not regain the ability to walk independently, even after rehabilitation $[11,12]$. It is thus important to develop rehabilitation strategies that will promote walking recovery after stroke.

Current best practice guidelines recommend that individuals with stroke should engage in early rehabilitative training that is intensive, repetitive, and task-specific to improve mobility and walking $[13,14]$. However, it can be challenging to reach this guideline for individuals with more severe stroke; the amount of walking practice achieved during rehabilitation is especially low for individuals requiring more assistance from their therapist to stand and walk [15]. Electromechanical devices such as body weight-supported treadmills and treadmill-based robotic devices have been proposed to provide walking practice to non-ambulatory individuals during stroke rehabilitation [16, 17], though some research has not supported their use $[18,19]$. A possible reason for the mixed findings is the suggestion that treadmill-based assisted gait training does not fully replicate the taskspecificity of overground walking [20].

Powered robotic exoskeletons are a more recent technology developed to enable walking for anyone with lower extremity weakness, without the constraints of prior mechanical devices. These wearable robots strap around the torso and legs to control joint motion to automate overground walking and can be used independent of a treadmill or overhead harness system. Early research has demonstrated safe use of powered robotic exoskeletons for individuals with stroke, but few clinical trials have been conducted to determine the efficacy of using such devices; fewer still have compared exoskeletal gait training to standard physical therapy care during early stroke recovery and rehabilitation [21-23]. Additionally, no studies have yet explored the perception and experience of either individuals with stroke or physical therapists towards using powered robotic exoskeleton technology for rehabilitation, a necessary consideration when introducing technology into practice [24].

The present study is designed to examine the adoptability of a powered robotic exoskeleton for stroke rehabilitation by determining the efficacy and acceptability of exoskeleton-based gait retraining. More specifically, this mixed-methods trial aims to: 1 ) determine the efficacy of exoskeleton-based gait rehabilitation to improve walking ability, function, cognition, and quality of life; as well as 2) explore the experience and perception of using a powered robotic exoskeleton for rehabilitation from the perspective of patients with stroke and their physical therapists. It is hypothesized that exoskeleton-based gait rehabilitation will result in greater improvements in walking ability, function, cognition, and quality of life compared to usual physical therapy care.

\section{Methods}

This nested mixed-methods study will be comprised of a multi-center, parallel-group randomized, controlled trial (RCT) with an embedded qualitative study [25]. A flow diagram of the study procedures can be seen in Fig. 1 . The methods for the quantitative and qualitative components are described here separately.

\section{Quantitative component: randomized, controlled trial Setting}

The RCT will be conducted at three rehabilitation hospitals, GF Strong Rehabilitation Centre (Vancouver, Canada), Glenrose Rehabilitation Hospital (Edmonton, Canada), and Parkwood Institute (London, Canada). Participants will be recruited from each respective inpatient stroke rehabilitation units over a period of up to three years.

\section{Participants}

Consecutive patients with subacute stroke admitted at each rehabilitation hospital will be identified by their treating physiatrists and therapists to be screened for eligibility by a member of the research team. Individuals will be included if they: 1) are within 3 months of stroke 


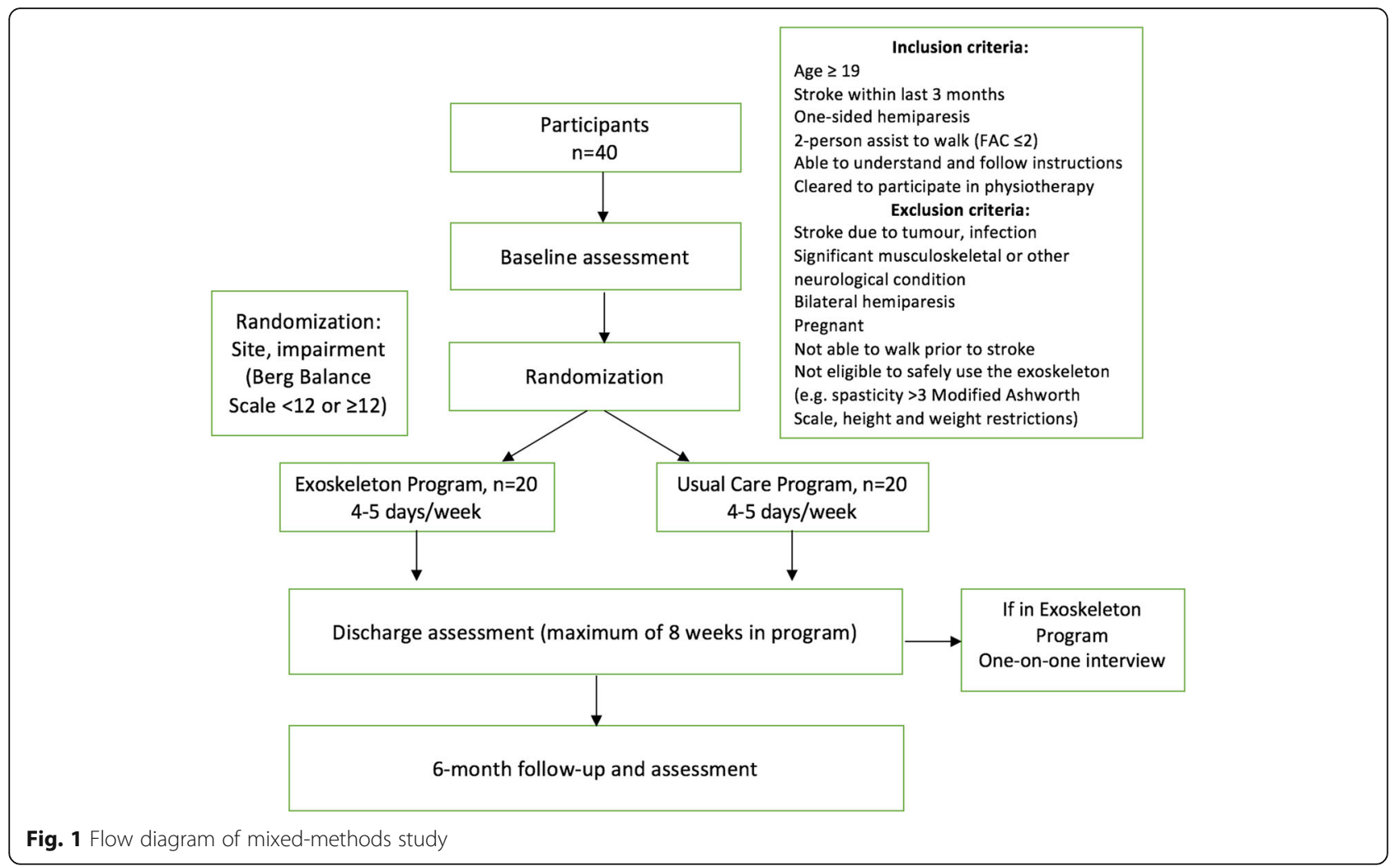

onset (ischemic infarct or intracerebral hemorrhage); 2) have one-sided hemiparesis; 3) are 19 years of age or older; 4) are able to understand and follow directions in English; 5) are able to communicate (verbal or physical yes/no indication); 6) are cleared to participate in physical therapy; and 7) require significant assistance (maximal assistance from one or two people) to walk. Individuals will be excluded from the study if they have: 1) a significant musculoskeletal or other neurological condition; 2) cardiovascular contraindications to exercise; 3) co-morbidities that would preclude activity; 4) or pain which is intolerably worsened with exercise. Individuals will also be excluded if they have any contraindications to using the robotic exoskeleton (pregnant, height/weight restrictions). The site coordinator at each hospital with obtain informed consent from potential trial participants.

\section{Randomization}

Participants will be randomized by the site coordinator after the baseline assessment at a one-to-one ratio to either the Exoskeleton group or Usual Care group using an online, third-party, permuted block randomization service (www.randomize.net, Interrand Inc., Ottawa, ON). As such, randomization will remain concealed until group allocation. Randomization will be stratified by site, to control for differences in standard of care (e.g., frequency and duration of physical therapy treatment, length of stay, rehabilitation and admission discharge timing, etc.). Participants will also be stratified by physical function, as baseline functioning is an independent predictor of outcomes such as community mobility and discharge destination $[8,26]$. Specifically, participants will be stratified using their baseline Berg Balance Scale score at enrolment, as it has been shown to be correlated with improved walking ability after robot-assisted gait training [27]. A cut-off score of 12 will be used to stratify participants, based on a study [28] which identified a score of 12 at rehabilitation admission to be predictive of regaining unassisted walking after four weeks.

\section{Exoskeleton device}

The EksoGT powered robotic exoskeleton (Ekso Bionics, Richmond, California, USA) will be used to provide the experimental intervention for this study. This exoskeleton has bilateral motor-actuated joints at the hip and knee, as well as a spring-loaded articulation at the ankle to support toe-off and foot clearance during gait via a footplate. The EksoGT is able to power the user's lower limbs in a walking pattern autonomously (without any active participation by the user), as well as with varied assistance to accommodate any force contribution by the user. The device software allows the therapist to control the degree of assistance, the parameters of gait (step height, step length, swing speed, etc.) and the automaticity of walking (how each step is triggered). These settings can be programmed to tailor the gait training to 
the individual to ensure active participation that is appropriately challenging. Guidelines for programming the device software to progress gait training with respect to robotic assistance are available in Additional file 1. The device does not provide balance support, and so the user is responsible for maintaining balance and shifting their weight appropriately.

\section{Exoskeleton intervention (experimental)}

Participants in the Exoskeleton group will have $75 \%$ of their standard physical therapy sessions replaced with exoskeleton-based gait rehabilitation. For example, 3 out of 4 weekly physical therapy sessions, or $45 \mathrm{~min}$ out of every 60-min session, will be dedicated to the exoskeleton intervention, reserving $25 \%$ of therapy time to be dedicated towards other goals. Participants in the Exoskeleton group will wear a powered robotic exoskeleton for their physical therapy sessions beginning after the baseline assessment to allow for repetitious stepping and walking practice from early in their rehabilitation stay. Training will be safely progressed, as tolerated, to reduce the amount of assistance provided by the exoskeleton and to increase the duration of continuous walking bouts. Guidelines for training progression can be seen in Table 1, and specific device programming is available in Additional file 1.

An algorithm will guide clinicians in deciding when to discontinue daily exoskeleton training (Fig. 2), as it has been found that therapist-guided overground walking practice is equally or more effective than electromechanically-assisted gait for improving walking function once people with stroke are ambulatory $[18,19]$. Once a participant reaches a functional threshold wherein they are able to walk for an extended period of time with only minimal assistance, therapists may begin to substitute daily exoskeleton training time to overground gait training. If a therapist chooses to fully discontinue use of the exoskeleton, they will still be required to focus on gait retraining for $75 \%$ of their weekly physical therapy time.

\section{Usual care intervention (control)}

Participants randomized to the Usual Care group will receive standard physical therapy care during their rehabilitation stay. Standard of care differs between sites, but typically involves 30-60 min physical therapy sessions, $4-5$ days a week. No specific instructions will be given to therapists providing therapy in the Usual Care group, except that they cannot use the robotic exoskeleton. Generally, physical therapy during stroke rehabilitation is provided with patient-specific goals in mind, and typically places a large focus on mobility and gait training. Participants in both the Exoskeleton and Usual Care group will be monitored twice a week using an activity tracker (activPAL3 micro, PAL Technologies, Glasgow, UK) to observe the amount of upright standing and walking performed in the physical therapy sessions per group.

\section{Evaluations}

All participants will be assessed at recruitment (baseline), at discharge or after 8 weeks of the intervention, and at 6 months by an assessor who is blind to group allocation. The exoskeleton intervention will be discontinued after 8 weeks, and standard physical therapy will be provided to all participants beyond 8 weeks for whom it is deemed appropriate by their care team.

\section{Primary outcome}

The primary outcome will be walking ability, measured using the Functional Ambulation Category (FAC) [29]. This is a 6-item scale designed to classify the level of physical support required by subjects in order to walk safely over $10 \mathrm{ft}$, extending from 1 (unable to walk without the assistance of two people) to 6 (independent walking overground on uneven surfaces and on stairs). It has been shown to have good test-retest reliability and validity in the hemiparetic stroke population [30]. The FAC is also responsive to change within the first four weeks post-stroke and up to six months post-stroke [30]; unlike other walking measures of speed or distance,

Table 1 Training progression for experimental group receiving exoskeleton intervention

\begin{tabular}{|c|c|}
\hline Timing & Exoskeleton training guidelines \\
\hline Week 1 (i.e. First 3-4 exoskeleton sessions) & $\begin{array}{l}\text { - Require } 30 \text { min of upright time in exoskeleton, no set requirement } \\
\text { for time in walking (expect approximately } 10 \text { min) } \\
\text { - Aim for at least } 250 \text { steps per session } \\
\text { - Familiarize with device, high assistance from therapist and robotics }\end{array}$ \\
\hline Week 2 (i.e. 5th session and on) & $\begin{array}{l}\text { - Require } 15 \text { min of walking time, of } 30 \text { min of upright time } \\
\text { - Aim for } 400 \text { steps per session } \\
\text { - Begin reducing assistance from therapist and robotics }\end{array}$ \\
\hline Week 3 (i.e. 10th session and on) & $\begin{array}{l}\text { - Require } 20 \text { min of walking time, of } 30 \text { min of upright time } \\
\text { - Aim for } 550 \text { steps per session } \\
\text { - Continue reducing assistance from therapist and robotics }\end{array}$ \\
\hline Week 4 and beyond (i.e. beyond 15 sessions) & $\begin{array}{l}\text { - Require } 25 \text { min of walking time, of } 30 \text { min of upright time } \\
\text { - Aim for } 700+\text { steps per session } \\
\text { - Minimal assistance from therapist and robotics }\end{array}$ \\
\hline
\end{tabular}




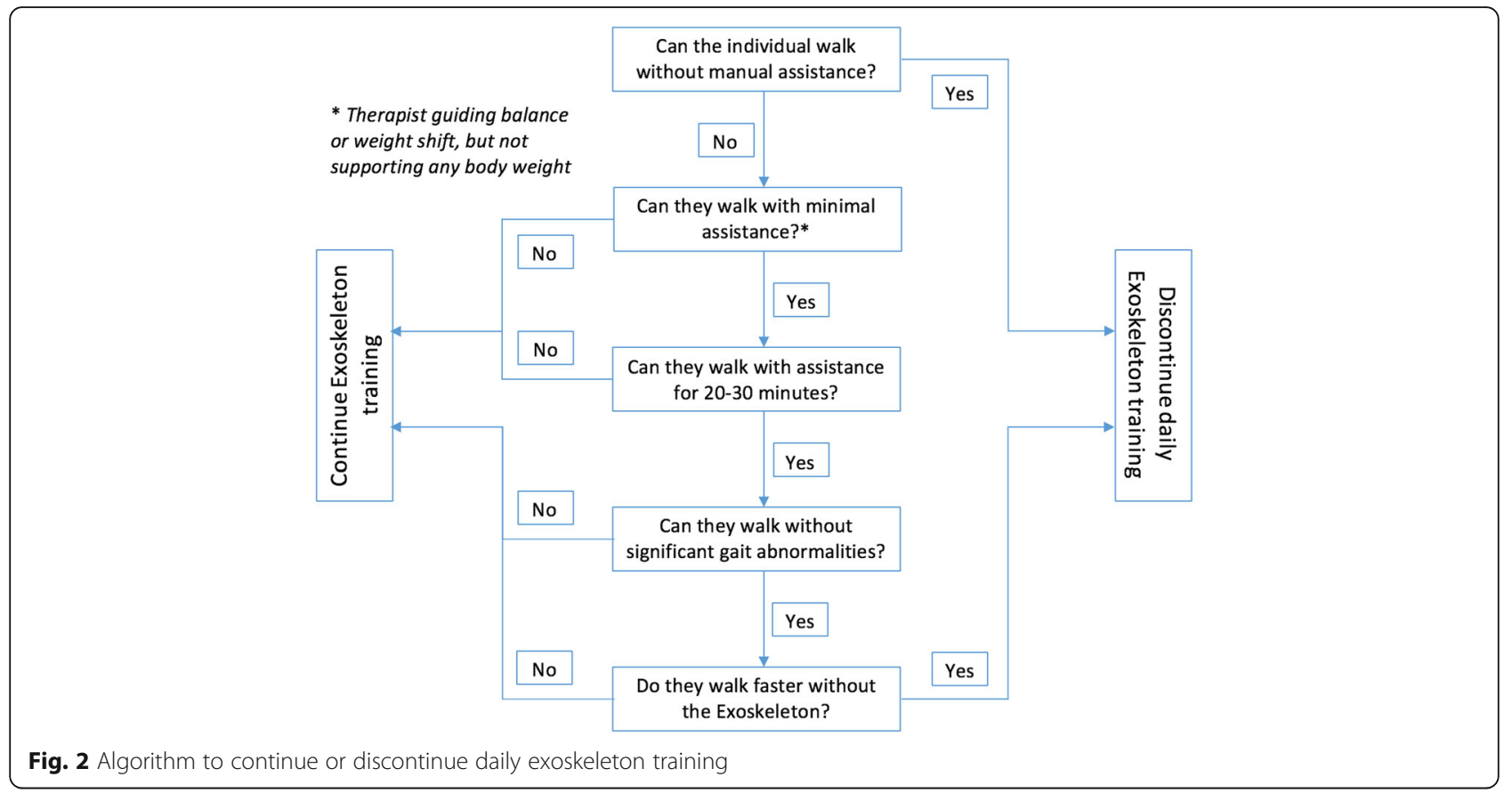

a value is assigned for the FAC even if the participant is not yet independent in walking.

\section{Secondary outcomes}

The secondary outcome measures will assess stroke impairment, walking performance (speed, endurance, daily step count), balance, cognition, and quality of life. The secondary outcome measures and schedule of data collection are listed in Table 2.

\section{Safety monitoring}

All sites will report minor and serious adverse events that occur from baseline through to the 6-month followup. Two expert physiatrists will review reports outlining adverse events, if they arise, annually, to advise on trial continuation.

\section{Sample size estimates}

A total of 20 participants will be enrolled in each group (total same $n=40$ ). This sample size was calculated using Stata Software (version 11, StataCorp, USA) and assumes a 2-point between-group difference in the Functional Ambulation Category at the end of the intervention [40], setting power at $80 \%$ and level of significance at 0.05 (2-sided). This calculation also assumes a standard deviation of 2.0 based on stroke inpatient FAC data from a study by Mehrholz et al. [30]. This betweengroup difference is realistic, given that participants are 2-person assist (score of 1 on the FAC) on enrolment, and it is expected that those in the Exoskeleton group will make greater improvements in walking ability (independent) compared to those receiving standard care (assistance or supervision required).

\section{Statistical analyses}

Descriptive statistics will be used to summarize data. An analysis of covariance (ANCOVA) will be performed to detect post-intervention differences between groups for the primary and secondary measures, using the respective baseline score as the covariate [41]. The significance level will be set at 0.05 , and all statistical tests will be two-tailed. Participant data will be analyzed on an intention-to-treat basis, and any missing data will be assessed and analyzed as appropriate using multiple imputation [42]. For measures without a baseline score (i.e., 5-Metre Walk Test, 6Minute Walk Test), an analysis of variance (ANOVA) will be employed. Confidence intervals (95\% CIs) will be reported, where applicable.

\section{Qualitative component: interpretive description}

This qualitative descriptive study will be conducted concurrently with the randomized controlled trial to determine the acceptability of the exoskeleton device for stroke rehabilitation from the perspective of patients with stroke and their physical therapists. The qualitative description methodology is useful when a straight description of phenomena is required, without the need for developing theory [43]. It is a rigorous methodology that provides a comprehensive summary of experiences and perceptions that is often used in health sciences research [43]. The qualitative design was informed by the COREQ (Consolidated criteria for reporting qualitative research) 
Table 2 Schedule of data collection

\begin{tabular}{|c|c|c|c|c|}
\hline Study Procedures & Screening & Baseline evaluation & Post-intervention evaluation & Six-month evaluation \\
\hline Timepoint & $-\mathrm{T}_{1}$ & $\mathrm{~T}_{0}$ & $\mathrm{~T}_{1}$ & $\mathrm{~T}_{2}$ \\
\hline Informed consent & + & & & \\
\hline Inclusion/exclusion criteria & + & & & \\
\hline Demographics & & + & & \\
\hline Randomization & & + & & \\
\hline \multicolumn{5}{|l|}{ Primary outcome measure } \\
\hline Functional Ambulation Category [30] & & + & + & + \\
\hline \multicolumn{5}{|l|}{ Secondary outcome measures } \\
\hline \multicolumn{5}{|l|}{ Impairment } \\
\hline Fugl-Meyer Assessment (Lower extremity) [31] & & + & + & + \\
\hline \multicolumn{5}{|l|}{ Functional } \\
\hline 5-Meter Walk test [32] & & & $(+)$ & $(+)$ \\
\hline 6-Minute Walk test [33] & & & $(+)$ & $(+)$ \\
\hline Berg Balance Scale [34] & & + & + & + \\
\hline activPAL mean step count (in PT) & & & $t^{a}$ & \\
\hline Days to unassisted ambulation & & & $+^{\mathrm{a}}$ & \\
\hline activPAL daily step count over 4 days $[35,36]$ & & & & + \\
\hline \multicolumn{5}{|l|}{ Cognitive } \\
\hline Montreal Cognitive Assessment [37] & & + & + & + \\
\hline \multicolumn{5}{|l|}{ Quality of life } \\
\hline Patient Health Questionnaire [38] & & + & + & + \\
\hline 36-Item Short Form Survey [39] & & + & + & + \\
\hline Adverse events screen & & & $t^{\mathrm{a}}$ & + \\
\hline
\end{tabular}

PT: Physical therapy.

() Parentheses indicate that the outcome will be assessed if the participant is able to walk without physical assistance.

andicates that the measure will be taken or monitored throughout the intervention period

checklist [44], which will be used to report the qualitative findings.

\section{Approach}

This study will be based in a postpositivist paradigm [45], assuming that exoskeleton users will have individual yet relatively patterned experiences and perceptions of the device. According to this paradigm, conclusions that are drawn regarding the acceptability of using a powered robotic exoskeleton can be generalized to other stroke rehabilitation sites regardless of the social contexts of the researcher and participants of this study.

\section{Participants}

Participants randomized to the Exoskeleton group in the quantitative RCT will be recruited from all sites to participate in qualitative interviews. All participants who undergo at least five training sessions in the exoskeleton will be invited to participate, provided they are able to communicate fully (English proficiency, non-severe aphasia). It is expected that $10-15$ participants with stroke will be thus be eligible and interviewed, during which time data sufficiency will be reached and identified themes would not need to be adjusted by further data collected [46, 47].

All physical therapists who have been fully trained to use the exoskeleton device and who provide the intervention for the RCT will be invited to participate in qualitative interviews. Five to 10 physical therapists are expected to be eligible and participate.

\section{Procedures / data collection}

Individual semi-structured interviews will be conducted in a private office with participants with stroke as well as with physical therapists from the RCT. Interviews will last approximately $30 \mathrm{~min}$ and will be conducted by the same researcher, whether in-person or by telephone, to maintain consistency. Interviews will be audio-recorded.

The semi-structured interview guides were developed by the lead author and reviewed by two physical therapists and two physiatrists (Additional file 2). Development of the interview guides was informed by the Unified Theory of Acceptance and Use of Technology (UTAUT) [48]. Questions explore user perspective towards the usage 
(fitting, duration, frequency, etc.) and utility (efficacy, perceived benefits, drawbacks, etc.) of the device.

\section{Data processing and analysis}

All interviews will be transcribed verbatim and analyzed using thematic analysis [49]. Transcripts will be read and re-read to develop ideas and interpretations about recurring, converging, and contradictory patterns. Once familiarized with the transcripts, raw data will be inductively coded by two investigators, then iteratively conceptualized into broad categories which will eventually be grouped into relevant themes to provide an understanding of how the exoskeleton is perceived by patients and therapists who use the device.

\section{Trustworthiness}

Drawing from the postpositivist criteria of credibility, transferability, dependability, and confirmability [50], various strategies will be employed to ensure the trustworthiness of this qualitative component of the study. Triangulation of multiple perspectives towards the exoskeleton by interviewing both individuals with stroke and physical therapists will promote the credibility of the qualitative findings. Furthermore, combining two research methods is another method of triangulation which will add depth and rigor to the study. The qualitative findings will provide context for the interpretation of the quantitative trial results.

Research reflexivity will support the transferability of the qualitative findings. By ensuring that the positioning of the authors and personal assumptions are accounted for in conducting the study and made known in reporting, readers will be able to determine the extent to which the findings can be generalized to their own context [50]. A reflexive journal will be kept in order to facilitate reflection on any assumptions, power differentials, and interpersonal dynamics that may arise during interviews that may influence data collection and analysis, which pertains to the dependability and confirmability of the qualitative methods [50].

Finally, negative case analysis and participant checking will enhance the credibility of the qualitative analysis. By exploring divergent perspectives during interviews and paying attention to opinions counter to the majority during analysis, we will develop a greater depth and understanding of the experience of using a powered exoskeleton for stroke rehabilitation. Bringing findings back to participants to ensure the analysis corresponds to their original account will help to ensure richness and accuracy of the findings. Synthesized analyzed data and resulting themes will be presented to participants in a document, written in non-scientific wording. Participants will be asked if the findings match their experience, and if they would like to change or add anything; any added data will be cross-referenced with existing codes and integrated into the analysis [51].

\section{Trial status}

Participant recruitment began on 5 May 2017 for GF Strong Rehabilitation Centre, on 7 December 2017 for Glenrose Rehabilitation Hospital, and on 8 August 2018 for Parkwood Institute. Participant recruitment is ongoing and projected to be completed by December 31, 2020 .

\section{Discussion}

This mixed-methods study is the first to investigate the adoptability of an exoskeleton device in stroke rehabilitation by concurrently determining the efficacy and acceptability of an exoskeleton-based gait retraining program during early stroke recovery. At a time when powered robotic exoskeletons are continually being developed, refined, and manufactured, the findings of this study will provide guidance to clinicians as to whether such devices should be employed for early stroke rehabilitation, and for which outcomes.

Currently, there are only a handful of inpatient rehabilitation facilities across Canada that house a powered robotic exoskeleton for clinical or research purposes. We anticipate that recruitment may be affected by potential participants' perception of the robotic device; some participants may decline participation because of the novel and intimidating nature of integrating robotics into treatment, while others excited for the device may be disappointed if they are randomized to the Usual Care group and may subsequently withdraw from the study. To account for these concerns, potential participants will be informed of the safety features and specific purpose of using the exoskeleton for the study, and those randomized to the Usual Care group will be offered an opportunity to trial the exoskeleton after their intervention period.

Compared to other studies of electromechanical devices and robot-assisted gait training in which the robotic intervention is rigorously performed several times a week for the entire duration of the intervention period, the current study presents a more realistic clinical intervention in which the exoskeleton use is integrated within standard physical therapy care and the frequency of exoskeleton use is reduced once a certain target in walking improvement is reached. Previous research showed that ambulatory individuals with stroke fare worse when confined to robotic or harness systems [17, 18], and thus our protocol will progress participants to activity without the robot once they are able. We anticipate that this method of exoskeletonuse will be more acceptable to therapists, as the algorithms presented for progressing the exoskeleton training or discontinuing use of the exoskeleton allows independence and clinical reasoning on the part of therapists. We also 
anticipate this will support a smooth translation of the research findings into clinical practice once findings are disseminated.

By also conducting qualitative interviews with participants and their therapists, a deeper understanding of the utility and potential limitations of powered robotic exoskeletons in today's health services for stroke rehabilitation will be gained alongside the efficacy findings. Without the positive reception of the device, regardless of demonstrated effectiveness, new technology often goes unused [20, 52]. Furthermore, the rich data gained from exploration of participant and therapist experience of using an exoskeleton will be integrated with the quantitative findings to serve knowledge translation efforts at study completion, as the personal accounts will potentially elucidate how best to utilize the device in therapy with respect to timing, frequency, set-up, and duration.

This study has several limitations. Individuals with more severe stroke requiring greater assistance to walk often have other impairments or co-morbidities that may affect their prognosis, which may affect recruitment as well as the outcomes of this research study. Another limitation is the inability to blind the therapists or participants to the study intervention. Finally, there is a chance that the qualitative data may not reach saturation, given the number of eligible participants with this sample size.

\section{Supplementary information}

Supplementary information accompanies this paper at https://doi.org/10. 1186/s12883-020-1617-7.

Additional file 1. Guidelines for Progressing Participant in Exoskeleton Group.

Additional file 2. Qualitative Interview Guides.

\section{Abbreviations}

ANCOVA: Analysis of covariance; ANOVA: Analysis of variance; $\mathrm{Cl}$ : Confidence interval; COREQ: Consolidated criteria for reporting qualitative research; FAC: Functional Ambulation Category; PT: Physical therapy; RCT: Randomized, controlled trial; SD: Standard deviation

\section{Acknowledgements}

We would like to acknowledge staff at each participating site who have helped in obtaining ethics and operational approval for the study: Vickie Buttar, Sarah Caughlin, Andreea Cotoi, Chihya Hung, and Mitch Longval.

\section{Authors' contributions}

JJE led the acquisition of funding from the Heart and Stroke Foundation. DRL, WBM, JY, and JJE formulated the research question and protocol of the study. DRL prepared the first draft of the protocol paper, with contributions and editing from WBM, MD, RT, JY, and JJE to the subsequent drafts. All authors have read and approved the final manuscript.

\section{Funding}

The Heart and Stroke Foundation of Canada Grant in Aid (G-15-0009030) program was the funding body for the study. The funders had no role in study design, data collection, analysis, or preparation of the manuscript. Peer review for the trial protocol was provided by the funding body. Additional funding supporting the independent work of the authors is as follows: Dennis R Louie is supported by the Heart and Stroke Canadian Partnership for Stroke Recovery Trainee Award and Vanier Graduate Scholarship program. Dr. Mortenson's work is supported by a New Investigator Award from the Canadian Institutes of Health Research. Dr. Janice Eng's work is supported by the Canada Research Chair Program and a Canadian Institutes of Health Research Foundation Grant (FDN 143340).

\section{Availability of data and materials \\ Not applicable.}

\section{Ethics approval and consent to participate}

The study protocol has received ethical approval from: the Clinical Research Ethics Board at the University of British Columbia (H15-01339), Vancouver, British Columbia, Canada; the Health Research Ethics Board at the University of Alberta (Pro00071806), Edmonton, Alberta, Canada; and the Health Sciences Research Ethics Board at Western University (108618), London, Ontario, Canada. Operational approval has also been granted by: the Vancouver Coastal Health Research Institute, Vancouver, British Columbia, Canada; Alberta Health Services, Edmonton, Alberta, Canada; and Lawson Health Research Institute, London, Ontario, Canada.

All participants (individuals with stroke, physical therapists) will provide signed informed consent to participate in the RCT and qualitative study.

Consent for publication

Not applicable.

\section{Competing interests}

The authors declare that they have no competing interests with respect to the research, the exoskeleton device manufacturer, authorship, and/or publication of this article.

\section{Author details}

${ }^{1}$ Graduate Program in Rehabilitation Sciences, Faculty of Medicine, University of British Columbia, Vancouver, British Columbia, Canada. ${ }^{2}$ Rehabilitation Research Program, Vancouver Coastal Health Research Institute, 212-2177 Wesbrook Mall, Vancouver, BC V6T 1Z3, Canada. ${ }^{3}$ Department of Occupational Science and Occupational Therapy, Faculty of Medicine, University of British Columbia, Vancouver, British Columbia, Canada.

${ }^{4}$ Glenrose Rehabilitation Hospital, Alberta Health Services, Edmonton, Alberta, Canada. ${ }^{5}$ Parkwood Institute Research, Lawson Health Research Institute, London, Ontario, Canada. ${ }^{6}$ Parkwood Institute, St Joseph's Health Care London, London, Ontario, Canada. ${ }^{7}$ Schulich School of Medicine \& Dentistry, Western University, London, Ontario, Canada. ${ }^{8}$ Division of Physical Medicine and Rehabilitation, Faculty of Medicine, University of British Columbia, Vancouver, British Columbia, Canada. ${ }^{9} \mathrm{GF}$ Strong Rehabilitation Centre, Vancouver Coastal Health, Vancouver, British Columbia, Canada.

${ }^{10}$ Department of Physical Therapy, Faculty of Medicine, University of British

Columbia, 212-2177 Wesbrook Mall, Vancouver, BC V6T 1Z3, Canada.

Received: 18 December 2019 Accepted: 14 January 2020

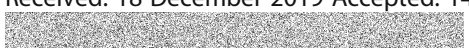

\section{References}

1. Lopez AD, Mathers CD, Ezzati M, Jamison DT, Murray CJL. Global and regional burden of disease and risk factors, 2001: systematic analysis of population health data. Lancet. 2006;367:1747-57.

2. Hankey GJ. Stroke. Lancet. 2017;389:641-54.

3. Feigin VL, Roth GA, Naghavi M, Parmar P, Krishnamurthi R, Chugh S, et al. Global burden of stroke and risk factors in 188 countries, during 1990-2013: a systematic analysis for the global burden of disease study 2013. Lancet Neurol. 2016;15:913-24.

4. Krueger H, Koot J, Hall RE, O'Callaghan C, Bayley M, Corbett D. Prevalence of individuals experiencing the effects of stroke in Canada: trends and projections. Stroke. 2015:46:2226-31.

5. Mayo NE, Wood-Dauphinee S, Ahmed S, Gordon C, Higgins J, McEwen S, et al. Disablement following stroke. Disabil Rehabil. 1999;21:258-68.

6. Portelli R, Lowe D, Irwin P, Pearson M, Rudd AG. Institutionalization after stroke. Clin Rehabil. 2005:19:97-108.

7. Bijleveld-Uitman M, Van De Port I, Kwakkel G. Is gait speed or walking distance a better predictor for community walking after stroke? J Rehabil Med. 2013:45:535-40. 
8. Pereira S, Foley N, Salter K, McClure JA, Meyer M, Brown J, et al. Discharge destination of individuals with severe stroke undergoing rehabilitation: a predictive model. Disabil Rehabil. 2014;36:727-31.

9. Bohannon RW, Andrews AW, Smith MB. Rehabilitation goals of patients with hemiplegia. Int J Rehabil Res. 1988;11:181-4.

10. Harris JE, Eng JJ. Goal priorities identified through client-centred measurement in individuals with chronic stroke. Physiother Canada. 2004;56:171-6.

11. Jørgensen HS, Nakayama H, Raaschou HO, Olsen TS. Recovery of walking function in stroke patients: the Copenhagen stroke study. Arch Phys Med Rehabil. 1995:76:27-32.

12. Shum ST, Chiu JKW, Tsang CPL, Wong CHP, Tsang RCC, Ma SL, et al. Predicting walking function of patients one month poststroke using modified rivermead mobility index on admission. J Stroke Cerebrovasc Dis. 2014;23:2117-21.

13. Billinger SA, Arena R, Bernhardt J, Eng JJ, Franklin BA, Johnson CM, et al. Physical activity and exercise recommendations for stroke survivors: a statement for healthcare professionals from the American Heart Association/American Stroke Association. Stroke. 2014;45:2532-53.

14. Hebert D, Lindsay MP, McIntyre A, Kirton A, Rumney PG, Bagg S, et al. Canadian stroke best practice recommendations: stroke rehabilitation practice guidelines, update 2015. Int J Stroke. 2016;11:459-84.

15. Rand D, Eng JJ. Disparity between functional recovery and daily use of the upper and lower extremities during subacute stroke rehabilitation. Neurorehabil Neural Repair. 2012;26:76-84.

16. Mehrholz J, Werner C, Kugler J, Pohl M. Electromechanical-assisted training for walking after stroke. What is the evidence so far? What have we learnt? Physiother. 2015;101:eS990-1.

17. Ada L, Dean CM, Vargas J, Ennis S. Mechanically assisted walking with body weight support results in more independent walking than assisted overground walking in non-ambulatory patients early after stroke: a systematic review. J Physiother. 2010;56:153-61.

18. Hidler J, Nichols D, Pelliccio M, Brady K, Campbell DD, Kahn JH, et al. Multicenter randomized clinical trial evaluating the effectiveness of the Lokomat in subacute stroke. Neurorehabil Neural Repair. 2009;23:5-13.

19. Duncan PW, Sullivan KJ, Behrman AL, Azen SP, Wu SS, Nadeau SE, et al. Body-weight-supported treadmill rehabilitation after stroke. N Engl J Med. 2011;364:2026-36.

20. Dobkin BH, Duncan PW. Should body weight-supported treadmill training and robotic-assistive steppers for locomotor training trot back to the starting gate? Neurorehabil Neural Repair. 2012;26:308-17.

21. Louie DR, Eng JJ. Powered robotic exoskeletons in post-stroke rehabilitation of gait: a scoping review. J Neuroeng Rehabil. 2016;13:53.

22. Postol N, Marquez J, Spartalis S, Bivard A, Spratt NJ. Do powered overground lower limb robotic exoskeletons affect outcomes in the rehabilitation of people with acquired brain injury? Disabil Rehabil Assist Technol. 2019:14:764-75.

23. Goffredo M, Guanziroli E, Pournajaf S, Gaffuri M, Gasperini G, Filoni S, et al. Overground wearable powered exoskeleton for gait training in subacute stroke subjects: clinical and gait assessments. Eur J Phys Rehabil Med. 2019 https://doi.org/10.23736/S1973-9087.19.05574-6.

24. Turchetti G, Vitiello N, Trieste L, Romiti S, Geisler E, Micera S. Why effectiveness of robot-mediated neurorehabilitation does not necessarily influence its adoption. IEEE Rev Biomed Eng. 2014;7:143-53.

25. Zhang W, Creswell J. The use of "mixing" procedure of mixed methods in health services research. Med Care. 2013;51:e51-7.

26. Frank $M$, Conzelmann $M$, Engelter S. Prediction of discharge destination after neurological rehabilitation in stroke patients. Eur Neurol. 2010;63:227-33.

27. Kim SJ, Lee HJ, Hwang SW, Pyo H, Yang SP, Lim MH, et al. Clinical characteristics of proper robot-assisted gait training group in nonambulatory subacute stroke patients. Ann Rehabil Med. 2016:40:183-9.

28. Louie DR, Eng JJ. Berg balance scale score at admission can predict walking suitable for community ambulation at discharge from inpatient stroke rehabilitation. J Rehabil Med. 2018;50:37-44.

29. Holden MK, Gill KM, Magliozzi MR, Nathan J, Piehl-Baker L. Clinical gait assessment in the neurologically impaired.Reliability and meaningfulness. Phys Ther. 1984;64:35-40.

30. Mehrholz J, Wagner K, Rutte K, Meißner D, Pohl M. Predictive validity and responsiveness of the functional ambulation category in hemiparetic patients after stroke. Arch Phys Med Rehabil. 2007;88:1314-9.
31. Park EY, Choi YI. Psychometric properties of the lower extremity subscale of the Fugl-Myer assessment for community-dwelling hemiplegic stroke patients. J Phys Ther Sci. 2014;26:1775-7.

32. Fulk GD, Echternach JL. Test-retest reliability and minimal detectable change of gait speed in individuals undergoing rehabilitation after stroke. J Neurol Phys Ther. 2008;32:8-13.

33. Fulk GD, Echternach JL, Nof L, O'Sullivan S. Clinometric properties of the six-minute walk test in individuals undergoing rehabilitation poststroke. Physiother Theory Pract. 2008;24:195-204.

34. Blum L, Korner-Bitensky N. Usefulness of the berg balance scale in stroke rehabilitation: a systematic review. Phys Ther. 2008:88:559-66.

35. Duncan PW, Sullivan KJ, Behrman AL, Azen SP, Wu SS, Nadeau SE, et al. Protocol for the Locomotor experience applied post-stroke (LEAPS) trial: a randomized controlled trial. BMC Neurol. 2007;7:39.

36. Haeuber E, Shaughnessy M, Forrester LW, Coleman KL, Macko RF. Accelerometer monitoring of home- and community-based ambulatory activity after stroke. Arch Phys Med Rehabil. 2004:85:1997-2001.

37. Toglia J, Fitzgerald KA, O'Dell MW, Mastrogiovanni AR, Lin CD. The minimental state examination and Montreal cognitive assessment in persons with mild subacute stroke: relationship to functional outcome. Arch Phys Med Rehabil. 2011;92:792-8.

38. de Man-van Ginkel JM, Gooskens F, Schepers VPM, Schuurmans MJ, Lindeman E, Hafsteinsdóttir TB. Screening for poststroke depression using the patient health questionnaire. Nurs Res. 2012;61:333-41.

39. Hopman WM, Verner J. Quality of life during and after inpatient stroke rehabilitation. Stroke. 2003:34:801-5.

40. Perry J, Garrett M, Gronley JK, Mulroy SJ. Classification of walking handicap in the stroke population. Stroke. 1995;26:982-9.

41. Twisk J, Bosman L, Hoekstra T, Rijnhart J, Welten M, Heymans M. Different ways to estimate treatment effects in randomised controlled trials. Contemp Clin Trials Commun. 2018;10:80-5.

42. Barnes SA, Lindborg SR, Seaman JW. Multiple imputation techniques in small sample clinical trials. Stat Med. 2006:25:233-45.

43. Sandelowski M. Whatever happened to qualitative description? Res Nurs Health. 2000;23:334-40.

44. Tong A, Sainsbury P, Craig J. Consolidated criteria for reporting qualitative research (COREQ): a 32-item checklist for interviews and focus groups. Int J Qual Heal Care. 2007;19:349-57.

45. Creswell JW. Qualitative inquiry and research design: choosing among five approaches. 3rd ed. Thousand Oaks: SAGE Publications; 2013.

46. Saunders B, Sim J, Kingstone T, Baker S, Waterfield J, Bartlam B, et al. Saturation in qualitative research: exploring its conceptualization and operationalization. Qual Quant. 2018;52:1893-907.

47. Hennink MM, Kaiser BN, Marconi VC. Code saturation versus meaning saturation. Qual Health Res. 2017;27:591-608.

48. Venkatesh V, Morris MG, Davis GB, Davis FD. User acceptance of information technology: toward a unified view. MIS Q. 2003;27:425-78.

49. Braun V, Clarke V. Thematic analysis. In: Cooper H, Camic PM, Long DL, Panter AT, Rindskopf D, Sher KJ, editors. APA handbook of research methods in psychology, Vol 2: research designs: quantitative, qualitative, neuropsychological, and biological. Washington: American Psychology Assocation; 2012. p. 51-71.

50. Morrow SL. Quality and trustworthiness in qualitative research in counseling psychology. J Couns Psychol. 2005;52:250-60.

51. Birt L, Scott S, Cavers D, Campbell C, Walter F. Member checking: a tool to enhance trustworthiness or merely a nod to validation? Qual Health Res. 2016;26:1802-11.

52. Langan J, Subryan H, Nwogu I, Cavuoto L. Reported use of technology in stroke rehabilitation by physical and occupational therapists. Disabil Rehabil Assist Technol. 2018;13:641-7.

\section{Publisher's Note}

Springer Nature remains neutral with regard to jurisdictional claims in published maps and institutional affiliations. 